\title{
An Aid System for Autonomous Mobility of Visually Impaired People on the Historical City Walls in Lucca, Italy
}

\author{
Massimiliano Donati*, Fabrizio Iacopetti, Alessio Celli, Roberto Roncella \\ and Luca Fanucci \\ Dept. Information Engineering, University of Pisa, Via Caruso 16, I-56122 Pisa (Italy) \\ \{massimiliano.donati, fabrizio.iacopetti, alessio.celli, roberto.roncella, \\ luca.fanucci\}@unipi.it
}

\begin{abstract}
The research project "The Walls for All" (in Italian "Le Mura per Tutti") aims at the realization of an aid system for visually impaired people, promoting autonomous mobility in a free time/cultural outdoor scenario. The selected installation site is a $4 \mathrm{~km}$ pedestrian and bike wide lane on top of the historical walls of Lucca city, Tuscany, Italy. The proposed system guides the user on predefined tracks (virtual path), realized by means of buried cables, that can be detected with the help of the Smart Cane, a traditional white cane equipped with custom electronics; and a mobile device featuring an ad-hoc developed Android application that provides geo-referenced guidance and context information. The system has been conceived, designed and successfully tested by visually impaired people of the Italian Association for the Blind and Visually Impaired - Section of Lucca.
\end{abstract}

Keywords: mobility aid system; visually impaired people; autonomous outdoor mobility; guidance system; smart white cane; GPS; Android app.

\section{INTRODUCTION}

According to the Italian Institute of Statistics (ISTAT) [1], the number of visually impaired people in Italy is 350.000 and, among them, the number of blind people is 60.000. In Europe visually impaired people are about 30 million [2].

For many people with visual impairments, autonomous mobility is often limited to the well-known home environment or to the surroundings, due to the dangerousness of moving alone: from obstacles encountered on the path (e.g. stairs, 
holes, etc.) to the danger represented by motor vehicles, subways, etc.. However, having some possibility of autonomous mobility beyond a limited context may improve the overall quality of life of these people, favoring relationships and increasing self-esteem.

People with visual impairments, and in particular blind people, needs to acquire some specific skills before moving autonomously outside a well-known context. Moreover, the use of mobility aid devices to recognize the current position on a route and to detect and avoid obstacles and dangers is often required. Before walking alone on a route, it is generally needed to have built a mental map of the route, enriched with reference points such as trees, building corners, lamps, sidewalks, stairs, etc., which can help the person to recognize his/her position.

Because of the difficulties and dangers involved in autonomous mobility, many people with visual impairments have often to give up moving alone.

The present work describes the continuation of the research project "The Walls for All" (in Italian "Le Mura Per Tutti") [3] and its current outcomes. The goal of the project is the realization of a mobility aid system for visually impaired people in a public outdoor place of free time, tourist and cultural interest. The project aims at promoting autonomous mobility on a predefined safe path installed on the historical walls of Lucca city, Tuscany, Italy. The developed solution provides the user with guidance and context information by means of a modified traditional white cane equipped with embedded electronics (Smart Cane), buried cables identifying the safe path (virtual path), and a specific Android application running on a mobile device. The project is being carried out at the Department of Information Engineering, University of Pisa, Italy.

Hereafter, Section 2 reports the state-of-the-art analysis of mobility aid systems. The proposed mobility solution and the installation site are respectively described in Section 3 and Section 4. Finally, conclusions are drawn in Section 5.

\section{STATE OF THE ART IN THE FIELD OF MOBILITY AIDS FOR VISUALLY IMPAIRED PEOPLE}

In the field of mobility aids for visually impaired users, different solutions have been proposed until now. The most recent solutions rely on modern ICT technologies and different working principles, while some others are traditional and well-known (e.g. white cane, dog guides, etc.).

Each proposed solution typically addresses some specific aspects and requirements of autonomous mobility. For example, the detection of obstacles in front of the user, the provision of navigation information to reach the desired destination, the tactile feedback as a mean of guidance on a route, etc.. The different proposed solutions have their own advantages and drawbacks.

The current section illustrates the main categories of mobility aid systems for people with visual impairments, providing some examples of ICT-based solutions that have reached the commercial or pre-commercial stage, and citing also some in progress or finished research projects [4]. 


\subsection{GPS-based geo-localization and guidance}

This category of mobility aid systems relies on geo-localization and guidance information based on the current user's position and on a map. Such systems exploit the GPS (Global Positioning System) signal and in some cases also terrestrial enhancement signals aiming at improving positioning accuracy [4]-[7]. Guidance information provided to the user are usually vocal messages and/or vibration feedback.

GPS-only based solutions rely on GPS positioning accuracy. In outdoor scenarios, the minimum accuracy is in the order of one/few meters, but near buildings, trees, etc., accuracy can raise up to ten meters or more. Such accuracy is therefore not completely suitable for mobility of visually impaired pedestrians, especially in an urban scenario, where routes are complex and present several obstacles (e.g. sidewalks, traffic lamps, etc.) and dangers (e.g. vehicles, stairs, etc.). Moreover, GPS-based systems cannot be used inside buildings due to the lack of the GPS signal coverage.

Two main sub-categories of GPS-based systems exist: i) applications for mobile devices and ii) stand-alone dedicated devices.

Among applications for mobile devices, an example is represented by the Android app Corsair [8]. Similar apps are available for different operating systems, as an example the application BlindSquare for iOS [9]. Other examples are available in literature [10].

Corsair is a GPS-based Android app for pedestrian navigation. The application provides the user with information about navigation, Points of Interest (POI) in the surroundings, ordered by category, and can also provide information about public transports (if available). The user is guided by means of vocal messages, or via the vibration of the mobile device that helps the user follow the right direction. The application offers other features that are particularly suitable for people with visual impairments, for example the repetition of the last played messages by pressing the earbud push-button, the provision of messages about the current position, etc.. A screenshot of the Corsair application is shown in Figure 1.

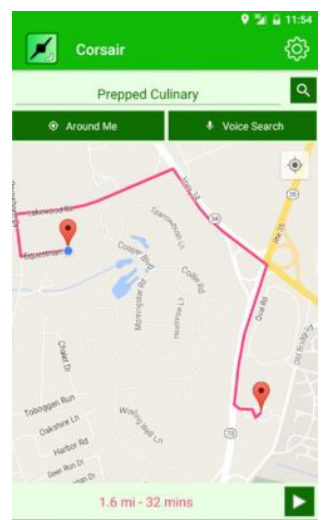

Figure 1. A screenshot of the Corsair application for Android mobile devices 
The application uses the same maps of other consumer services, but provides an ad-hoc realized user interface. The application exploits the mobile and/or WiFi data communication infrastructure to download the needed maps. No other infrastructure delimiting or identifying the path is needed. The application is freely available to users and is Open Source.

Within the sub-category of GPS stand-alone dedicated devices, Trekker Breeze Plus [11], shown in Figure 2, is a commercial device for pedestrian navigation of visually impaired people. Along the route, the device provides vocal messages concerning street names, the presence of crosses and information on nearby POIs such as public buildings and shops. The device also provides step-by-step instructions on how to use it.

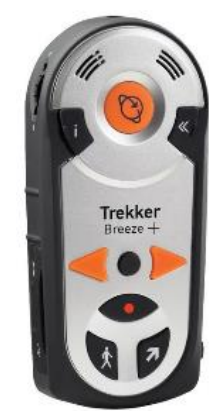

Figure 2. The stand alone GPS-based navigation device Trekker Breeze Plus

On the producer's website it is not explicitly mentioned whether the terminal exploits data communication networks to retrieve the needed maps, or if maps are pre-loaded or have to be uploaded to the device in other ways (as an example via a personal computer and an Internet connection). The system does not need any infrastructure delimiting or identifying the path.

Other similar commercial devices exist [10], some of them providing also very specific user interfaces like Braille Note GPS that features a Braille interface [12].

Finally, there are several research projects that led to the development of prototypical GPS-based systems [13] [14].

\subsection{Obstacle detection}

Other mobility aid systems for visually impaired people aim at detecting obstacles in front of the user and warning him/her accordingly. Those systems are known as Local Obstacle Detection (LOD) systems and are designed to inform the user about the presence and possibly the position of obstacles.

Such systems are typically implemented by means of electronic devices that are mounted on a traditional white cane or integrated with it, or they are carried by users in their hands. The devices are equipped with detection sensors and provide a human-machine interface. The most used sensing technologies are ultrasounds, infrared and LASER [4] [15]. 
Anyway, currently available devices are not able to detect all obstacles in front of the user, mainly because of the very different geometrical and physical properties of obstacles (e.g. shape, composition, height from the ground, etc.).

Some research projects concerning obstacle detection and classification are focused on RADAR-based systems, as an example [16]. Until now, LOD systems based on RADAR technologies have not reached the commercial stage because the performance of such systems is still not adequate, mainly due to technical difficulties related to their design and miniaturization.

Among commercial LOD systems, Ultracane from BEL [17] is a white cane whose handle embeds ultrasound ranging sensors. The device emits ultrasound beam in different directions and processes the received echoes to obtain information about the presence and the distance of obstacles in front of the cane. Two pushbuttons/vibration actuators on the cane handle provide the user with information about obstacles by means of a vibration that is modulated according to the position of the obstacle. The device is able to detect obstacles on the ground and up to a height of about $1.5 \mathrm{~m}$. The device and the detection areas are shown in Figure 3.

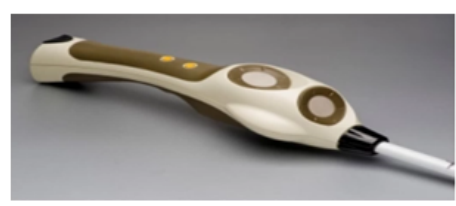

(a)
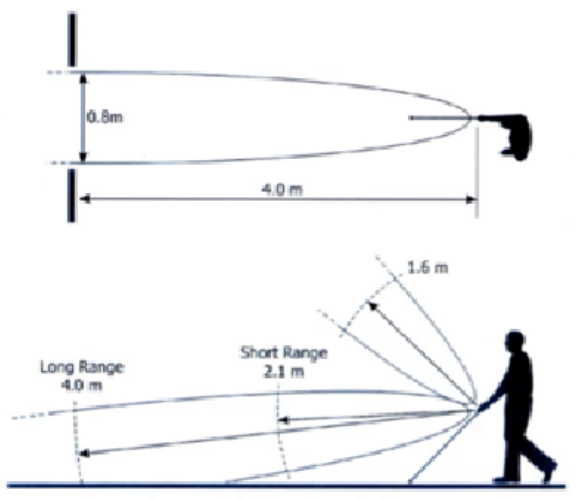

(b)

Figure 3. The Ultracane handle (a) and the illustration of the detection areas (b)

Ultracane does not require neither data communication networks nor the installation of any infrastructure on the path. The producer's website underlines the importance of a training phase of at least some days with an instructor to learn how to use the device properly.

Safewalk [18] is another example of LOD cane providing the user with information about the presence of obstacles in front of him/her via vibration feedback and audio messages. In particular, the user is warned about the danger of falling or stumbling due to changes of the ground level, both uphill and downhill, due to obstacles in front him/her and due to suspended obstacles: lamps, poles, stairs, cars, sidewalks, open blinds, etc.. The system, shown in Figure 4, is suitable for both outdoor and indoor environments. 


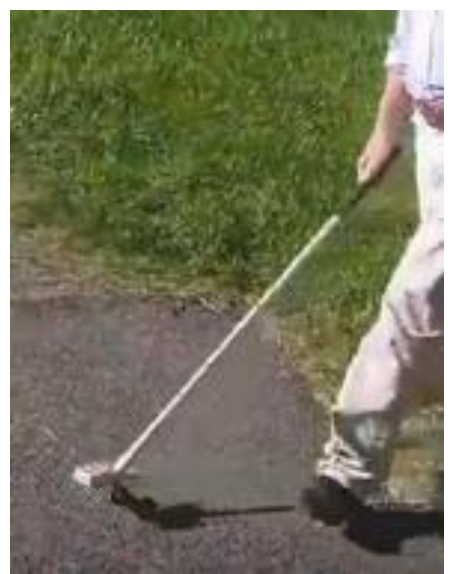

Figure 4. The prototypical version of Safewalk system

The lower-end of the cane is provided with a double wheel that allows the user to perceive the characteristics and roughness of the ground and to reduce the effort in keeping the cane. The system is made from aluminum in order to be light and sturdy. It is powered by a rechargeable battery that allows for about seven days of a typical usage without the need of recharging. Different settings are available, for example the minimum obstacle detection distance.

Safewalk does not use data communication networks and does not require the installation of infrastructures on the path. At the time of writing (July 2018), it is in pre-commercial stage, and the producer offers the possibility of booking the product.

Tom Pouce II (Pollicino), produced by BEL [19], is an example of LOD device to be mounted on the traditional white cane (see Figure 5). The detection approach used in the first version of the device (2011) is based on infrared technology. The detection distance range is selectable by the user by means of a 3-position slide switch. The device provides the user with information about the presence of obstacles via three different types of vibration: continuous vibration, $8 \mathrm{~Hz}$ - and 15 $\mathrm{Hz}$-modulated vibration, as schematically illustrated in Figure 6.

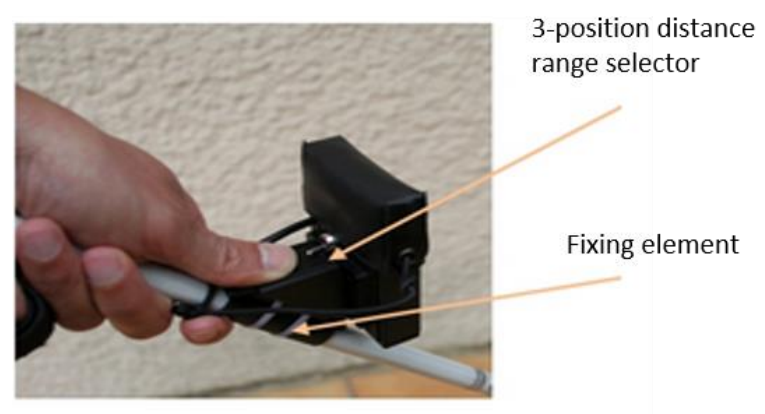

Figure 5. Tom Pouce II system installed on a traditional white cane 


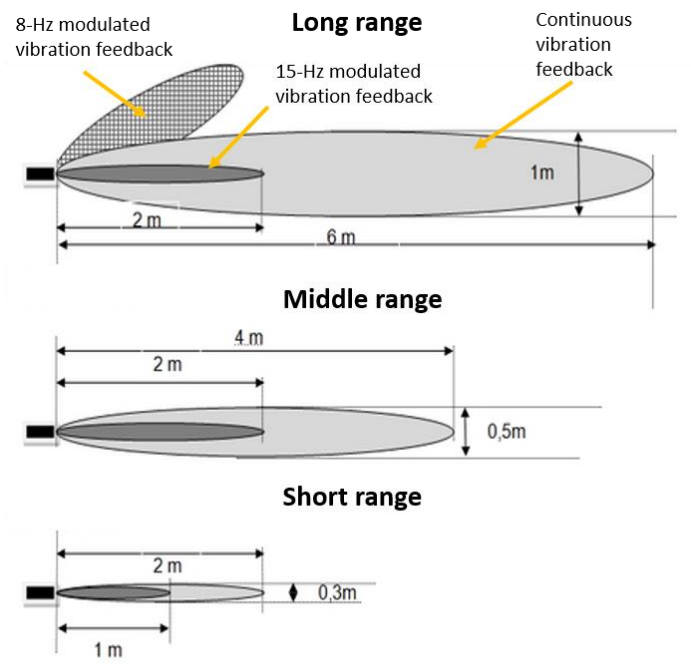

Figure 6. Tom Pouce II system: different types of vibration signalling obstacles in different areas

The modulation of the vibration informs the user about the distance and position of the obstacle: continuous vibration for far obstacles on the ground, $15 \mathrm{~Hz}$ modulated vibration for near obstacles on the ground, $8 \mathrm{~Hz}$-modulated vibration for near suspended obstacles. The terms "far" and "near" refers to the range of the sensing area selected by means of the slide switch.

On the website, the producer asserts that the complexity for the user in selecting the sensing distance and interpreting the vibration modulations, results in an improvement of the system usability providing a better obstacle awareness.

Tom Pouce II does not use the traditional data communication networks and does not require the installation of any infrastructure on the path. As it occurs with other similar devices, a training course of some days with an instructor is recommended to allow the user to learn using it.

An infrared obstacle detection device to be hold in the user's hand is Pollicino Light [20], produced by BEL and shown in Figure 7. The device is particularly suitable for indoor environments and in case the environment ground structure is known it may avoid the use of the white cane. The detection distance is selectable by means of a 2-way switch and the orientation of one side of the device in the hand with respect to ground (orienting the top or bottom side of the device towards the ground represents a 2-way selection). Four different ranging distances may be selected: $8 \mathrm{~m}, 4 \mathrm{~m}, 2 \mathrm{~m}, 30 \mathrm{~cm}$.

Pollicino Light does not use data communication networks and does not require the installation of infrastructures on the path. The device was a commercial product sold in 2014, but at the time of writing (July 2018) it was not possible to know its availability on the market. As with other similar systems, a training course was recommended to allow the user to use it effectively. 


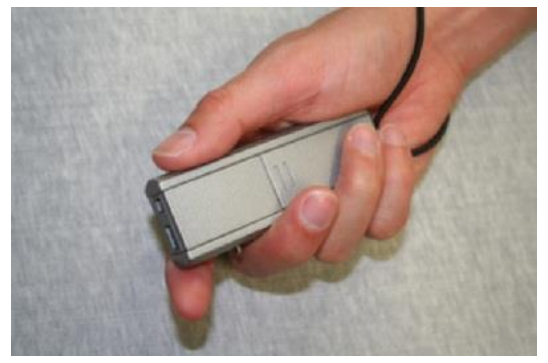

Figure 7. Pollicino Light system

Regarding research projects, the results of several activities in the field of LOD systems have been published in literature. For example, the system described in [21] is based on ultrasound obstacle detection, but there are many others exploiting different sensing technologies and working principles [4].

\subsection{Enhancement of the user's visibility}

Among mobility aid systems, some solutions aim at making users more visible to other pedestrians or to vehicle drivers, in order to improve safety in autonomous mobility.

An example of such systems is LetiSmart Luce [22], shown in Figure 8. It consists of a kit to be mounted on the bottom end of the white cane to allow for a high visibility in low lighting conditions.

LetiSmart Luce is essentially a battery powered lighting device, emitting light according to environmental lighting conditions. It is a commercial product and does not require neither data communication networks not the installation of an infrastructure on the path.

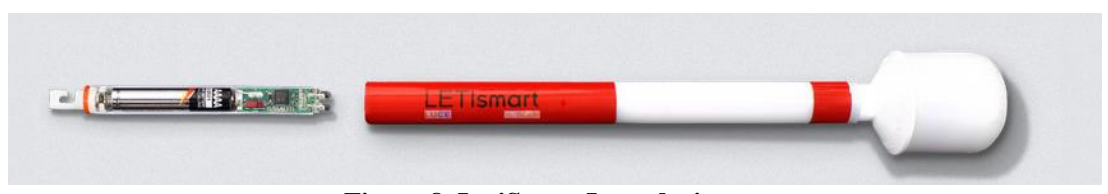

Figure 8. LetiSmart Luce device

In the future, it is foreseen the evolution of LetiSmart Luce into a new device integrated with the city public transport system and/or other city services that will provide users with the vocal information needed to move autonomously in the urban environment in the city of Trieste, Italy.

\subsection{Obstacle detection and environment recognition by means of vision systems}

A different category of mobility aid systems includes devices based on cameras mounted on helmets, glasses, etc. that analyze the surrounding environment and 
provide the user with vocal information and/or vibration feedback. Such system output aims at helping users to avoid obstacles on the path and at providing them with localization information and the perception of the surrounding environment and of nearby objects.

It is possible to find some vision systems at the prototypical or pre-commercial stage. Horus [23] is an example of vision system for objects and environment recognition that provides the user with vocal information. It consists of cameras mounted on a headset and of a portable processing unit, as shown in Figure 9.

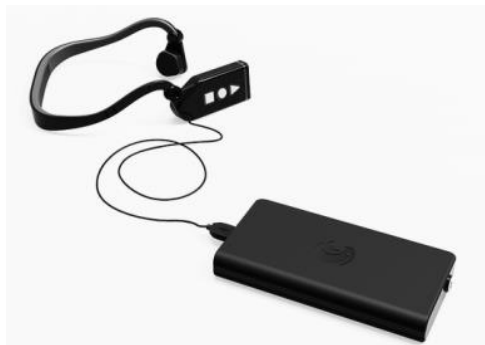

Figure 9. Horus system: cameras mounted on the headset and the portable processing unit

The system learns objects and faces during an initial training phase, then it is able to detect and recognize some objects and the face of known people. Once an object is recognized, Horus can help the user reach and grab the object via vocal information. Additionally, it can read the text on a book page, on a road sign, on a timetable, etc..

The user interacts with the system by means of some push-buttons on the headset and on the portable unit, which are easily identifiable by their different shapes. Using these push-buttons, the user can navigate the audio menu entries and activate the different device features. In some circumstances, Horus provides some vocal information automatically.

Horus is produced by Horus Technology. At the time of writing (July 2018), the system is in pre-commercial stage, and the producer offers the possibility of booking the product. From the information available on the producer's website, Horus seems not to require any data communication network (e.g. mobile and WiFi networks). The system does not require any specific infrastructure installed in the environment.

There are currently some other obstacle detection and environment recognition systems based on cameras and/or different kind of sensors [4]. For example, the device described in [24] exploits the fusion of Artificial Vision and GPS positioning while the one reported in [25] uses 3D Multisense technology for pedestrian crossing identification.

\subsection{User localization via signal reception and processing}

In this category of mobility aid systems, the user's position is determined by a portable unit that processes one or more types of signals received from transmitters 
placed in the environment and possibly also processing data read from kinematic sensors, as in Indoor Positioning Systems (IPS). Typical signals used are WiFi, Ultra Wide Band (UWB), Bluetooth, Infrared (IR), Light-Fidelity (LiFi). Once determined the position of the user, the localization information are typically provided via vocal messages or vibration feedback, often by means of a smartphone/tablet and a dedicated app.

Such systems are suitable for both outdoor and indoor environment. Indeed, the installation and management of a transmitter network is not always feasible in an outdoor environment.

These systems are usually the prototypical result of research activities, and only seldom they reached the state of commercial product.

An example of a commercial solution for indoor navigation exploiting signals from transmitters in the environment is the StepInside mobile app developed by Senion [26]. The application targets the general public, but could be of some use for visually impaired people.

The StepInside app, elaborating the signals received from WiFi transmitters and from Bluetooth beacons and the kinematic data provided by the smartphone sensors, localizes the user on a map of the building with one or even several floors. The working scheme of the user mobile terminal is shown in Figure 10.

The app is designed for indoor navigation in shopping centers, airports, etc., providing users with information on their position and location-based services.

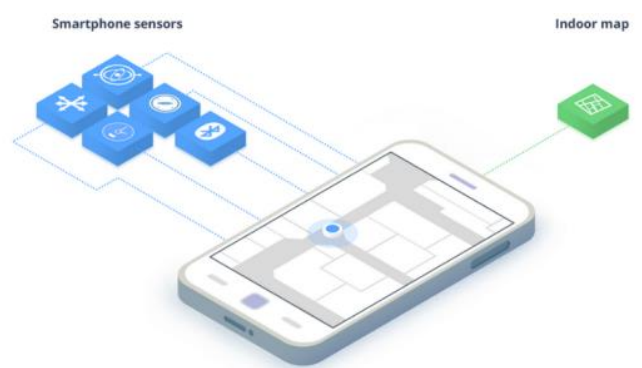

Figure 10. Representation of the working scheme of the user terminal in the IPS by Senion

The local communication/localization infrastructure can be partially exploited to provide other services (e.g. Internet access, etc.). The cost of the system (e.g. management system, map realization, etc.) is customer-specific and is therefore unknown.

There are currently some other guidance systems based on the reception and processing of different kinds of signals [4]. For example, the system developed in [27] exploits the modulation of the light emitted by LED bulbs to transmit LiFi data to a portable device that processes them and determines the position of the user inside mapped buildings. The system described in [28] is instead based on a white cane, Bluetooth and IR modules installed in the environment and a user device that receives IR signals, communicates with Bluetooth modules and provides location information to the user. 


\subsection{RFID-based guidance systems}

In recent years, guidance systems based on RFID technology were proposed. For example, the system defined in [29] uses a predefined safe path identified by means of buried RFID tags that are detected by a RFID reader mounted on a white cane. A mobile user terminal, connected to the reader, provides the user with location and navigation vocal information according to the RFID code read and a predefined online map. At the time of writing (July 2018), no updated information about the system may be found on the Internet, except for some news dated 2016 reporting some work in progress for the improvement of a previous prototypical system.

Another example is represented by Radio Frequency Identification Walking Stick (RFIWS) that was developed to help blind people navigate on the sidewalk [30]. The system helps the user in detecting and calculating the distance to the sidewalk border.

\subsection{Guidance systems based on guide tiles}

Predefined paths based on guide tiles [31][32] are often installed in many public places and buildings (e.g. railway stations, subways, squares, etc.). Guide tiles have different kinds of surface pattern that can be recognized and followed by the user using the tip of the white cane and/or with the feet. The different patterns signal the user the presence of a straight track, a curved one, the proximity to a sidewalk, etc.. Figure 11 shows some examples of tiles with grooves and reliefs patterns.
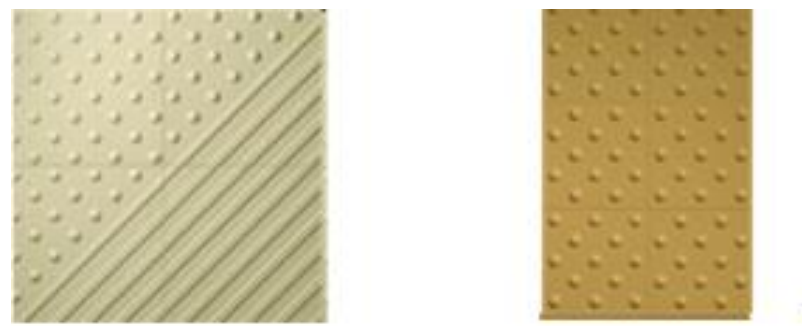

Figure 11. Some different patterns in Loges-Apice guide tiles

The installed paths allow the user to follow a safe route in an indoor and/or outdoor scenario (see Figure 12). However, such guidance system requires the user to obtain a preliminary knowledge of the route.

This guidance system may have a huge impact on existing buildings and places and has high installation costs. 


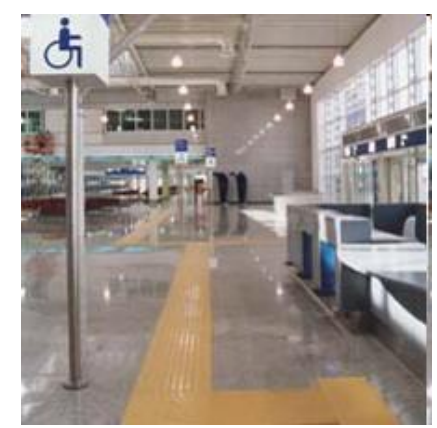

Figure 12. Loges-Apice path installed in a public building

\subsection{White cane}

The white cane is one of the most traditional mobility aid for visually impaired people. However, its use is not easy at all and specific training courses must be attended in order to learn how to use it for moving alone and for recognizing reference points, obstacles, etc.. Moreover, the white cane is not effective in all situations. For example, it does not allow to detect obstacles that are not lying on the ground (e.g. an open blind, etc.).

\subsection{Dog guides}

The well-known mobility aid represented by the dog guide is effective in many situations, but the interaction between the user and the dog poses some requirements that are not very easy to be managed. The user must in fact establish and maintain a strict, engaging and continuous relationship with the dog [33].

\section{THE PROPOSED SYSTEM}

The developed aid system addresses an outdoor urban mobility scenario, and in particular living an outdoor public place of tourist/cultural interest. In detail, the proposed system is focused on the provision of GPS-based guidance and context information on a predefined path and not on the obstacle detection approach on a whatever path. The advantage is that the selected path is quite safe, except for the still existing possibility of unforeseen obstacles (e.g. a bicycle that has been parked where it should not be, etc.). However, obstacle detection can be carried out by users via the developed Smart Cane just as if they were using a traditional white cane on their route.

Since the initial phase of the project, end-users' associations were actively involved in order to better fulfil user requirements. The collaboration started during the requirements definition and continued until prototype testing, carried out directly by some end-users. 
The main user requirements in the autonomous outdoor mobility scenario were defined in relation to the control of an electronic device and the kind and amount of information needed for an effective navigation.

The system, further described in the following sections, is schematically illustrated in Figure 13. The system architecture consists of three main elements:

- the virtual path;

- the Smart Cane;

- the mobile device with an ad-hoc Android application.

The guidance feature is realized combining two kinds of information. The first is the tactile vibration feedback produced by the Smart Cane when used on the predefined safe path (virtual path). The second one, provided by an Android app running on a mobile device, consists of vocal messages with navigation information and other information concerning the surrounding environment. Such vocal information is based on the GPS position of the user and on a predefined map for the specific installation site.

Tactile vibration feedback was chosen as the most suitable feedback type to signal the presence of the virtual path and, at the same time, to leave the hearing channel free. The hearing channel is very important for visually impaired people to locate themselves in the environment and to navigate, which is accomplished by detecting and analyzing the traffic noise, recognizing open spaces, etc.. For the same reason, the system provides vocal messages mainly on user's request. The content and the detail level of vocal messages were chosen addressing users' needs. In particular, vocal messages were aimed at helping the user build and follow a mental map of the path, providing information about reference points that can be identified along the route and that help the user have awareness of the current position.

The Smart Cane is connected to the mobile device through a Bluetooth link. The user can interact with the system via an 8-switch user interface mounted on the Smart Cane handle. The whole route is shortly described via a vocal help message that can be listened to before facing the route. While walking, the user can repeatedly query the system in order to obtain vocal information about nearby relevant points and the distance from them. In this way it is possible for him/her to locate himself/herself on the mental map of the environment he/she has built.

Vocal information may be listened to via earphone (Bluetooth or wired) or via device speakerphone, allowing for the use of the system with only one hand, as normally no interaction with the mobile device is required.

The mobile device with the Android application is not strictly needed for path detection. In such a case, the only information available is the vibration feedback that signals the presence and position of the virtual path.

The selected installation site was the pedestrian/bicycle lane of about $4 \mathrm{~km}$ on top of the historical walls of Lucca city, Tuscany, Italy. This route is very popular among people from Lucca and the surroundings, but also among tourists from other parts of Tuscany. From the lane it is possible to access green areas along the Walls, named "Bastioni" (bastions) or "Baluardi". 


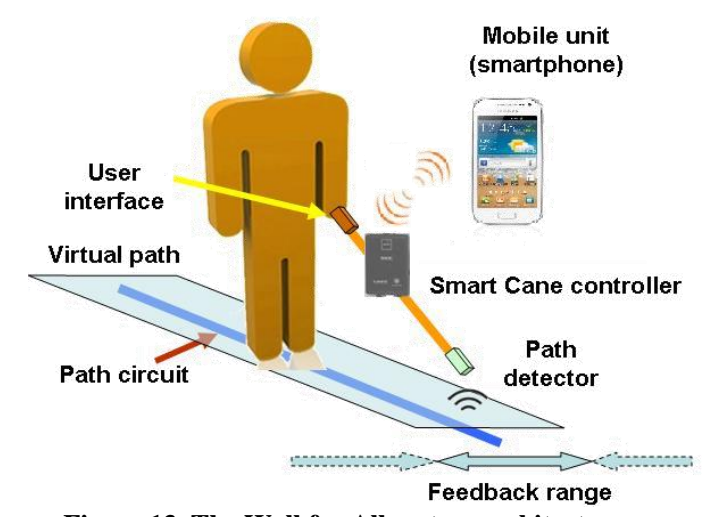

Figure 13. The Wall for All system architecture

\subsection{The virtual path}

The virtual path implements the predefined path that the user can walk and explore by means of the Smart Cane. The elements composing the virtual path are the following:

- tracks;

- $\quad$ branch points, where three or more tracks join together;

- $\quad$ points of interest along the path (e.g. benches, etc.).

Another kind of element mapped in the proposed mobility aid system is represented by points of interest far from the path (e.g. historical buildings, etc.). A schematic representation of the above mentioned elements is shown in Figure 14.

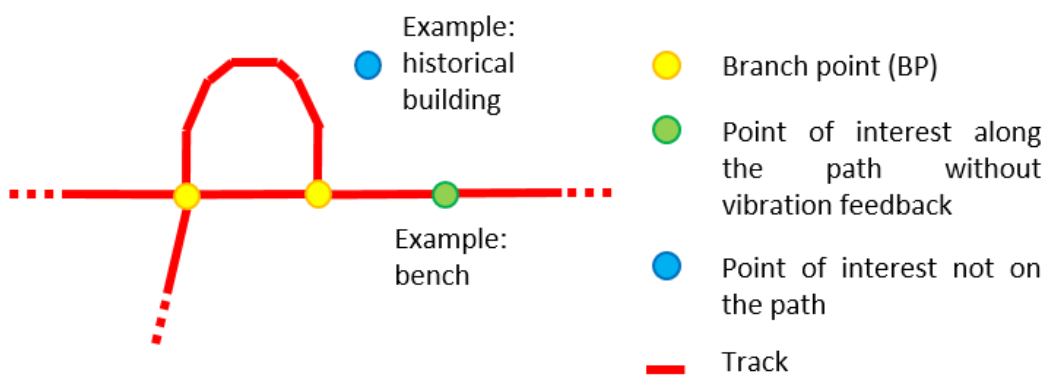

Figure 14. Elements mapped in the proposed mobility aid application

The tracks of the virtual path are realized by means of a couple of parallel electrical wires in which a modulated current, injected by a signal generator, generates a variable magnetic field that is detected by the path detector mounted near the Smart Cane tip. When the smart cane tip is within a range of about $0.25 \mathrm{~m}-0.5 \mathrm{~m}$ from the center of the track, the Smart Cane handle vibrates with an intensity roughly inversely proportional to the distance of the tip from the center of the track. 
The joining of three or more tracks represents a branch point. For a length of about $1 \mathrm{~m}$ starting from the joining point, the two cables of each track are replaced by a unique twisted cable, so that the magnetic field is heavily shielded and is not detected by the Smart Cane. In branch point areas, having therefore a diameter of about $2 \mathrm{~m}$, the Smart Cane provides no tactile vibration feedback.

Points of interest along the path are identified by the cancellation of the magnetic field for a length of about $1 \mathrm{~m}$, which is accomplished using a twisted cable in replacement of the two parallel cables.

Points of interest that are not along the path are not directly reached by the cable infrastructure. They are mapped simply by the GPS coordinates in the Android application and represent relevant cultural/outdoor points outside the walked route.

The placement of cables must occur at a mutual distance of about $3 \mathrm{~cm}$ (this value is anyway not critical). In case of a permanent installation of the virtual path infrastructure, as is the case for the walls of Lucca city, the cables can be buried under quite any kind of material up to a depth of about $5 \mathrm{~cm}-10 \mathrm{~cm}$, as illustrated in Figure 15. At the frequency used $(10 \mathrm{kHz})$, the magnetic field is not shielded by concrete/asphalt/ground soil, both wet and dry.

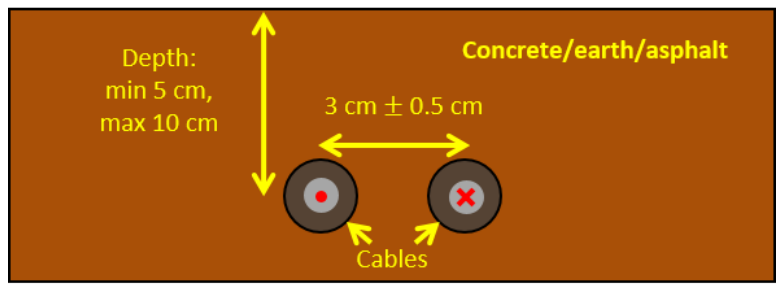

Figure 15. Schematic view of the cable placement

Another possible type of installation, not realized within the current project, is a surface installation on existing floors (e.g. below a carpet, etc.). In such a case, with minimal installation costs, the system may provide a guidance function (also without exploiting the GPS signal) also inside or around a public building, an exhibition, etc.

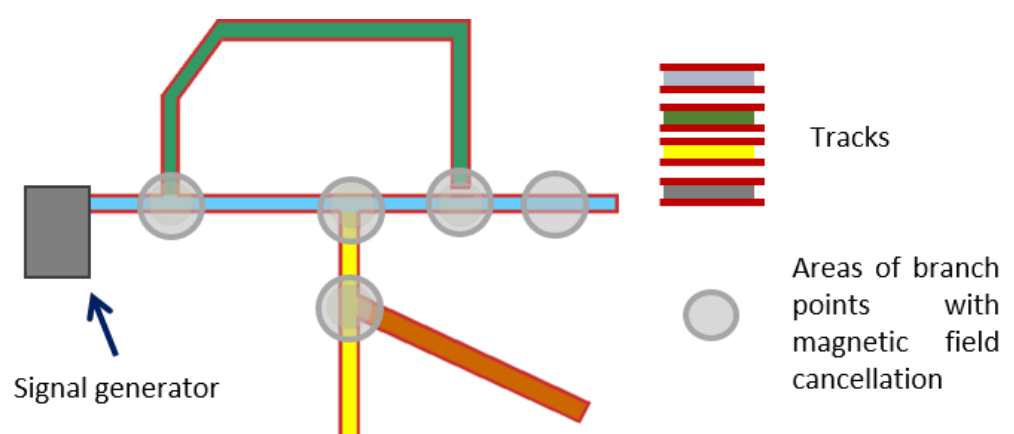

Figure 16. Virtual path circuit architecture 
The electrical circuit identifying the virtual path must be a closed loop (see Figure 16). A single signal generator is able to drive a path up to about $400 \mathrm{~m}$. For longer paths, it is necessary to use several circuits implementing sub-paths. Two or more loop circuits, driven by different signal generators, usually converge in branch points, where the magnetic field is strongly attenuated and therefore not detected; otherwise, detection issues may arise from the overlap of magnetic fields produced by currents from different generators that have unrelated phases and possibly slightly different frequencies.

Figure 17 shows the schematic view of the waveform of the current flowing within the cables; Figure 18 reports the scope view.

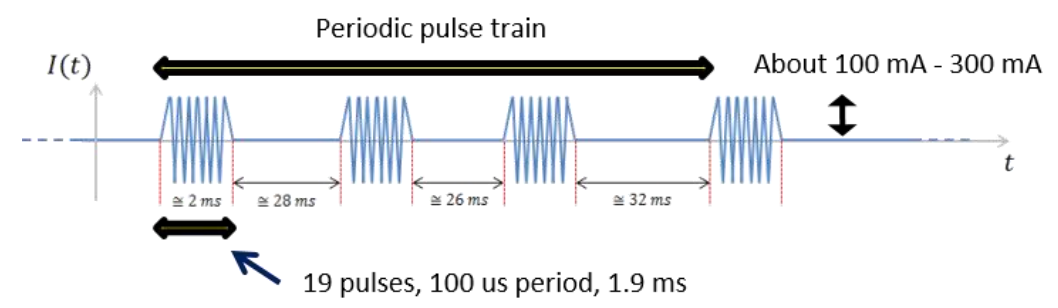

Figure 17. Schematic view of the current waveform in the loop circuit

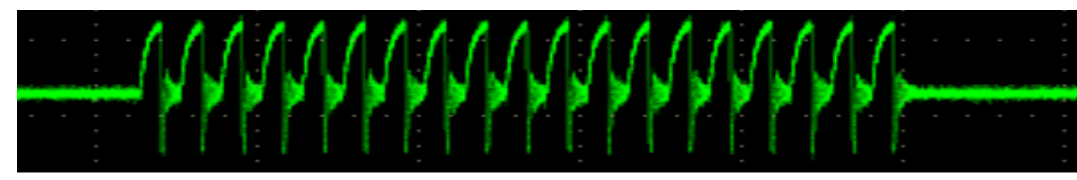

400 us/div, $200 \mathrm{~mA} /$ div

Figure 18. Scope view of the current waveform within the cables

Enabling or excluding existing path segments is quite easy and only requires a modification of cable connections within junction boxes. As long as a loop circuit does not exceed the maximum allowed length, the addition of new tracks is possible without the need to add new signal generators. It simply requires to make, in a junction box, the proper connection of the newly installed cables and the already existing circuit. In a similar way, for many of the points of interest with magnetic field cancellation and for some branch points it is possible to enable/disable the magnetic field by simply reconnecting in a different way the cables in some junction boxes (see Figure 19). In fact, in many points the two straight cables and the twisted cable were buried together in order to face possible modifications of the loop circuit, if required in the future. 


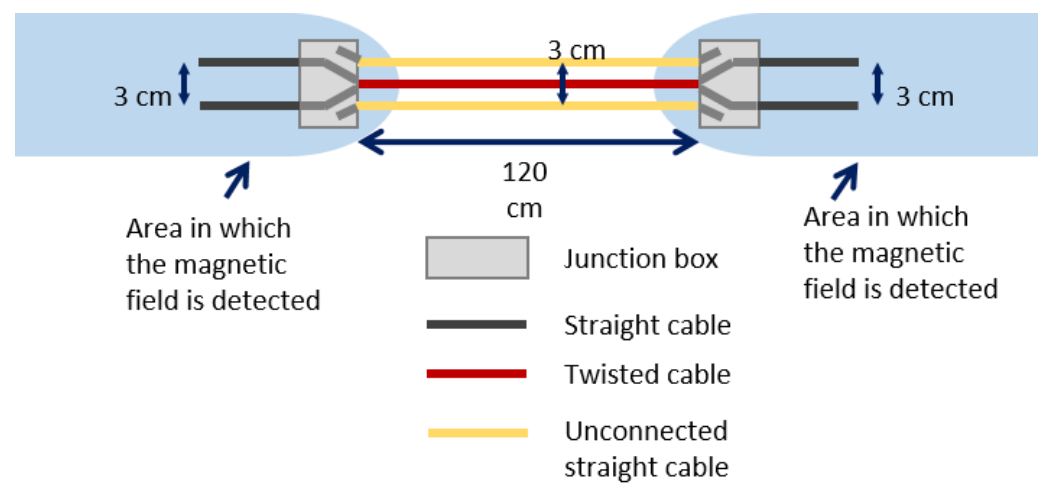

Figure 19. Installation details for a point of interest with magnetic field cancellation

The user can realize to have approached a relevant point with magnetic field cancellation, expected if he/she queries the system while walking on the route, when the vibration feedback stops.

In case of branch point, the joining tracks can be discovered by moving the Smart Cane in a circular area with a diameter of about $2 \mathrm{~m}$ around the point. The detailed information about joining tracks and where they lead to, provided by the application, can help the user to select the desired route.

Concerning points of interest on the path, they are typically related to benches placed within few meters aside from the track. Once the user has detected the short signal interruption, he/she can locate the bench using the smart cane as a traditional white cane, helped by the information provided by the application about on which side and how far it is located from the track.

Due to the relatively low GPS accuracy/precision, which is generally at least some meters or more in the installation site, branch points and points of interest with magnetic field cancellation are placed far from each other at a distance greater than about $15 \mathrm{~m}-20 \mathrm{~m}$, so that with the proposed system the GPS accuracy and precision do not represent a fundamental concern.

\subsection{The Smart Cane}

The Smart Cane is a fiberglass white cane equipped with some interconnected electronic devices and components developed within the project. In particular:

- the Smart Cane Controller (SCC);

- the Path Detector (PD);

- the input/output User Interface (UI).

The Smart Cane, further described hereafter, is shown in Figure 20 and its architecture is illustrated in Figure 21. 


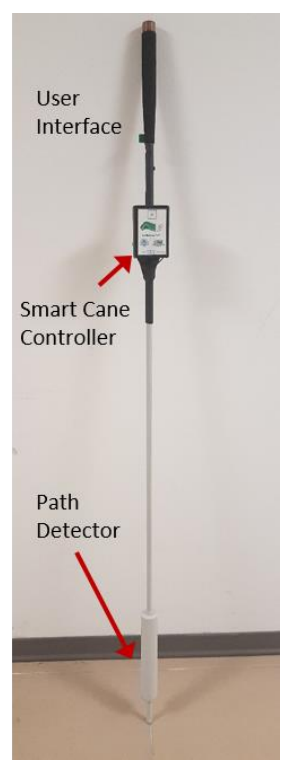

Figure 20. The Smart Cane

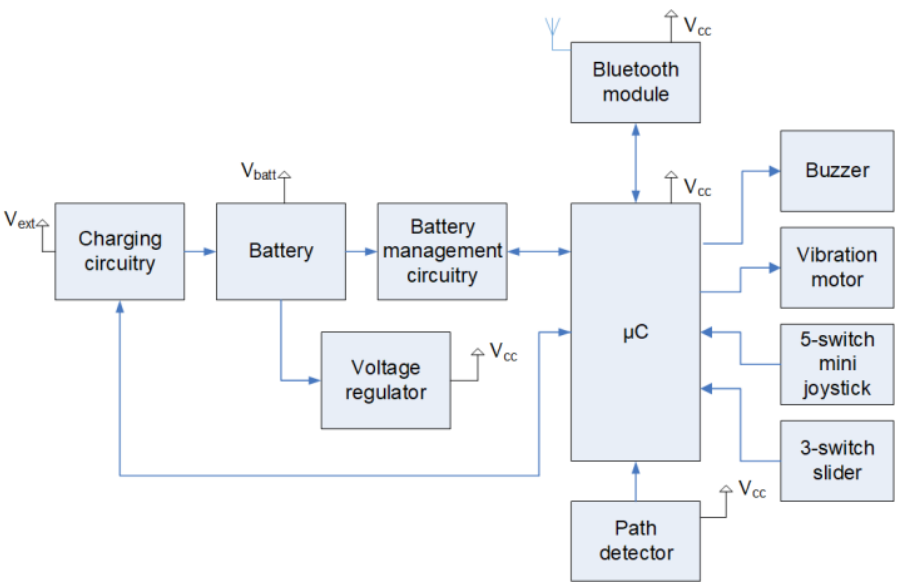

Figure 21. The Smart Cane Architecture

The input/output UI was designed and tested by final users during the first project phases. In Figure 22 some details of the user interface are illustrated. The input interface consists of a 5-switch mini-joystick, with Forward, Backward, Left, Right and Central controls, and of a 3-position slide switch, with Forward, Backward and Central controls. The mini joystick can be operated by means of the thumb finger and the slide switch by means of the index finger, without the need to lose the grip on the handle. The output UI includes a vibration motor installed in the cane handle and a buzzer. 
The Smart Cane Controller contains the main electronic subsystem of the Smart Cane. Its main components are an ATMega32L 8 bit microcontroller, which executes an embedded $\mathrm{C}$ compiled application with a code footprint of about $30 \mathrm{kB}$, a circuit for battery charging and monitoring, a Bluetooth module, a buzzer and a driver for the vibration motor.

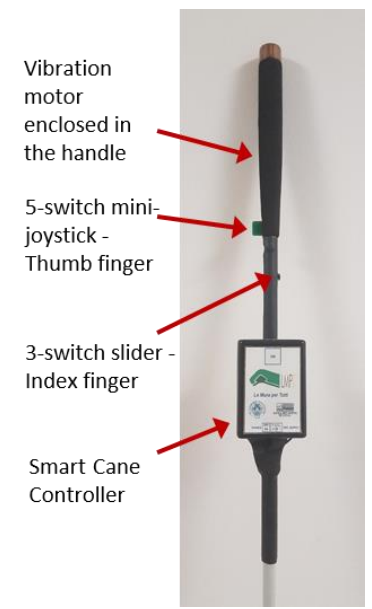

Figure 22. The Smart Cane User Interface (UI) and the Smart Cane Controller (SCC)

The SCC is connected to the input UI. Each button press/release events is sent to the mobile device via the Bluetooth link and allows the user to control most of the navigation and information functions of the Android application. For user convenience, the buzzer provides audio feedback about press and release events. Moreover, it also warns the user about the low battery condition.

The Path Detector is an electronic circuit (see Figure 23) mounted in proximity of the Smart Cane tip and enclosed in a cylindrical case.

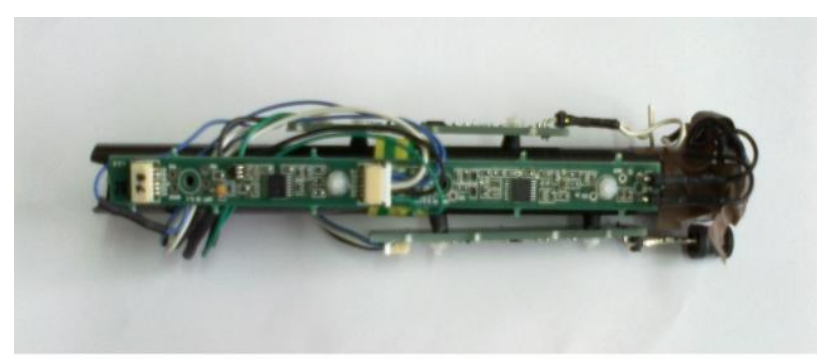

Figure 23. The Path Detector (PD)

The PD is connected to the microcontroller inside the SCC via a flat cable inside the cane. Within a range of about $25 \mathrm{~cm}-50 \mathrm{~cm}$ from the center of the virtual path cables, the output signal of the PD contains some pulses that are recognized by the SCC microcontroller as belonging to a valid pattern generated by the path magnetic 
field. Most spikes and other signals due to environmental electrical noise are rejected by the hardware and/or the firmware. Figure 24 reports the scope view of the current within the cables and the corresponding path detector output for a given distance from them. When the output signal of the PD is compliant with the expected parameters, the microcontroller activates a vibration motor in the cane handle, with an intensity roughly proportional to the path signal intensity and therefore inversely proportional to the distance from the center of the virtual path. By moving and/or sweeping the cane, the user can locate the side borders of the path and, approximately, its center.

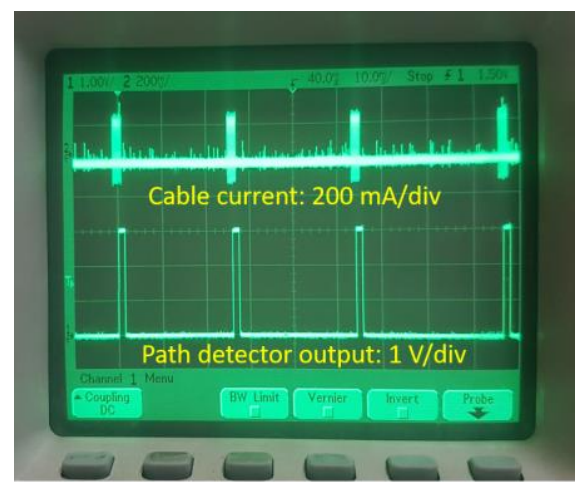

Horizontal division: $10 \mathrm{~ms} / \mathrm{div}$

Figure 24. Scope view of the current within the cables vs the path detector output signal

The average overall current draw of the different Smart Cane components is less than $100 \mathrm{~mA}$, and battery life is some hours in a typical scenario. Battery recharging may be performed via a common $6 \mathrm{~V}-500 \mathrm{~mA}$ mains power supply connected to a dedicated socket on the SCC.

\subsection{The mobile device and the Android application}

The third element of the system is a mobile device that runs an ad-hoc Android application. Its main task is to track the route of the user and to provide him/her synthesized audio messages when requested by means of the Smart Cane input UI.

The mobile device is required to be equipped with Android OS $\geq 4.0$ and ear piece with a mic. Also it has to feature an internal GPS receiver and a Bluetooth interface. No restriction are present on the device screen size and form factor (i.e. smartphones, tablet), nor constraint on memory and processing capabilities.

Considering the middle-range segment of Android devices, the built-in Bluetooth interface is able to manage multiple connections (i.e. to the smart cane and optionally to the earpiece) as required. The internal GPS receivers does not critically suffer from GPS shielding caused by user's body; therefore the mobile device could also be kept leaning to the body or in a pocket. Moreover, the precision 
of the GPS signal resulted to be generally sufficient in the test and installation site without particular filtering or corrections. In light of these considerations, common commercial devices are suitable for this kind of employment.

The application requires the privileges to operate with the GPS receiver and the Bluetooth interface of the device, as well as to access the phone manager in order to make call or sending SMSs in case of emergency. Additionally, online or preloaded offline text-to-speech voice database is required to play the messages.

Once the application has been launched, generally no interaction with the ondevice controls is needed because it is entirely controllable by means of the Smart Cane UI, and the device can be also locked.

Different kind of guidance/touristic message are available during the walk:

- overall description of the path, useful to build the mental map of the entire environment;

- $\quad$ name and distance of recently approached and nearby branch points and tracks joining in them, where they take to and which is the direction to be taken (e.g. left/right);

- name and distance of next points of interest on the path (e.g. benches, etc.);

- information about points of interest not on the path (e.g. the description of nearby historical buildings, etc.).

Even if not strictly required to use the system, the application features a graphical user interface, mainly developed for debug/demonstration purposes. Figure 25 shows the application running on a Samsung A3 smartphone.

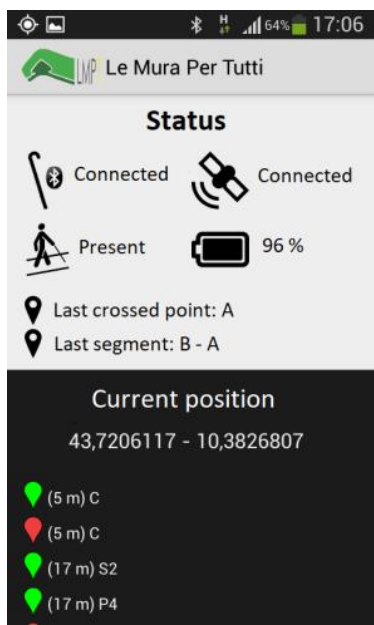

Figure 25. The main window of the Android application

The upper section reports the status of the connections with the Smart Cane and the GPS signal, the presence or absence of the virtual path signal and the Smart 
Cane battery level. The information about the last point and segment (couple of the last two points) visited by the user are also available. The lower section provides the coordinates of the current position of the user and the list of relevant points in increasing order of distance from such a point.

The application provides also a configuration window to allow the customization of the list of parameters reported in Table 1.

Table 1. Configuration and preference parameters of the App
\begin{tabular}{|l|l|}
\hline Parameter & Description \\
\hline $\begin{array}{l}\text { Smart Cane Bluetooth } \\
\text { address }\end{array}$ & $\begin{array}{l}\text { Selector of the Smart Cane Bluetooth among the } \\
\text { list of bonded devices }\end{array}$ \\
\hline Detection radius & $\begin{array}{l}\text { Distance in meters that makes the relevant point } \\
\text { to be considered as crossed in the application }\end{array}$ \\
\hline Emergency Phone Number & $\begin{array}{l}\text { Phone number to be called or to send to SMSs in } \\
\text { case of emergency during the walking }\end{array}$ \\
\hline Path log & $\begin{array}{l}\text { Storage of the entire path walked by the user using } \\
\text { Keyhole Markup Language (KML) }\end{array}$ \\
\hline Guidance Message Autoplay & $\begin{array}{l}\text { Autoplay of messages when the user reaches an } \\
\text { intersection point }\end{array}$ \\
\hline
\end{tabular}

The software architecture is shown in Figure 26. It consists of a series of cooperating modules that implements the desired functionalities: localize the user during the walk and provide information based on the current position and the a priori knowing of the relevant points position in the path.

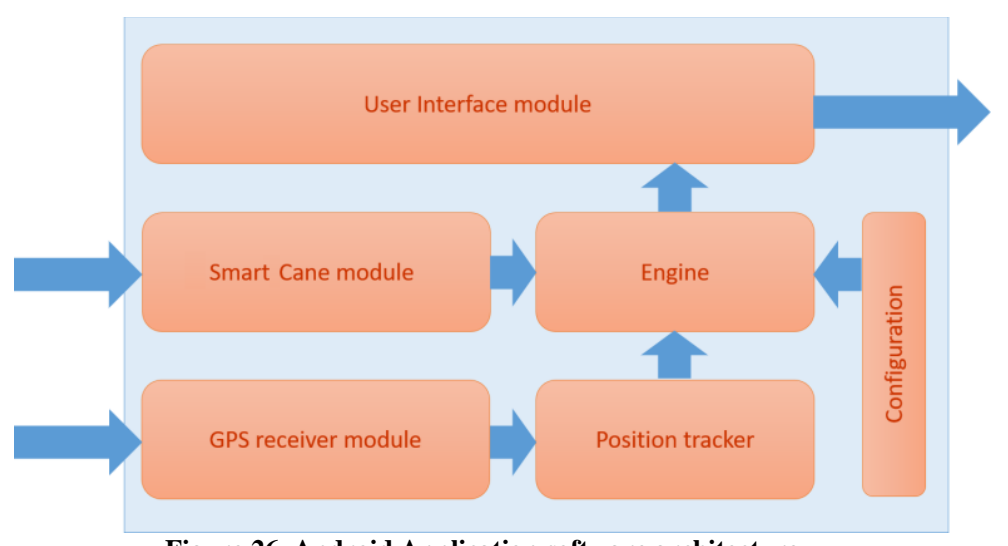

Figure 26. Android Application software architecture

The Smart Cane module runs in a dedicated thread. It manages the Bluetooth connection with the SCC in order to receive the commands given by the user and the updates of the virtual path detection status in every second. 
Also the GPS Receiver module runs in a threads. It reads in every second an updated position of the device from the GPS to localize the user in the space.

The Position Tracker module maintains the information about the current position CurrPos of the user (i.e. GPS coordinates) and implements a circular queue Quеие [10] for the automatic tracking of the last relevant points approached by the user during the walk.

The Engine module is the decision-maker module of the application. It uses the commands coming from the user and the position tracking information to select the messages to be played. Moreover, it monitors the status of the connections with the Smart Cane and the GPS receiver, providing service messages in case of faults, and finally it handles the refreshing of the user interface.

The user interface module is in charge of making available to the user the synthesized messages via built-in or externally connected audio output interfaces. Moreover, this module is responsible for the update of the information that appear on the main window when the device screen is unlocked.

The configuration contains the value of the parameters described in Table 1, that affects the behavior of the application, and the map of the entire path, loaded from an XML file, that represents the a-priori knowing of the installation environment In particular, the map is organized into three sections:

- An array Arr_path[N] containing the name and the GPS coordinates of all the relevant point along the virtual path (i.e. branch points and points of interest with signal cancellation);

- An array Arr_poi[M] containing the name, the detailed description and the GPS coordinates of all the relevant points in the installation environment of interest outside the virtual path;

- A matrix Mat[N][N], in which both columns and rows represents the relevant points along the virtual path and the elements are defined as follow:

- Mat $[i][i]$ contains the detailed description message for the i-th point.

- Mat $[i][j]$ contains the guidance message for the user that approaches the $\mathrm{i}$-th point coming from the $\mathrm{j}$-th point, if and only if a track between the two relevant points exists.

At the start-up of the application, the Engine loads the configuration and generates the SmartCane and the GPS receiver threads. After few seconds, when the Bluetooth connection with the Smart Cane Controller has been established and the GPS signal locked, a service message inform the user that the system is ready.

From this point on, the Position Tracker updates the CurrPos and the Queue variables using the coordinates provided by the GPS Receiver while the SmartCane module forwards the virtual path detection status to the Engine. In particular, a new relevant point $\mathrm{P}$ is marked as crossed, and put in the head position of the queue, if the distance between $\mathrm{P}$ and the CurrPos is less than the detection radius parameter:

$$
\text { Queue }[\text { head }]=P \text {, iff distance }(P, \text { CurrPos }) \leqslant \text { detection radius }
$$


When the user queries the system by means of the Smart Cane joystick, the Engine provides the proper message to the output user interface based on i) the current position, ii) the virtual path detection status, iii) the queue of the last crossed relevant points and iv) the map of the path in the configuration.

In particular, the Engine firstly orders the arrays of relevant points by distance from the user's position and calculates the target relevant point assuming no inversion of direction:

$$
\begin{gathered}
\text { Ordered_Arr_path }=\text { order(Arr_path, CurrPos }) ; \\
\text { Ordered_Arr_poi }=\text { order(Arr_poi, CurrPos }) ; \\
\text { Target_point }=\text { target(Ordered_Arr_path, Queue[head]); }
\end{gathered}
$$

Then, depending on the activated control button, the requested message is played:

- Joystick - Right button: the short names and distances of the two closest relevant points along the virtual path (Ordered_Arr_path[0].name; Ordered_Arr_path[1].name)

- Joystick - Left button: the short names and distances of the two closest points of interest ouside the virtual path (Ordered_Arr_poi[0].name; Ordered_Arr_poi[1].name)

- Joystick - Up button: the information about the current position of the user. In particular:

O if the user is on the virtual path, the app provides the short name of the closest target branch/interest point the user is moving to (Ordered_Arr_path[target].name);

0 if the virtual path is not detected and the user is within a branch/interest point area, the app provides the name of this relevant point (Queue[head].name);

○ otherwise an "out of path" service message is provided.

- Joystick - Down button: the detailed information on nearby or recently approached branch and interest points. In particular:

○ if the virtual path is detected, the app provides the detailed information (short name and description) of the nearest target branch/interest point the user is moving to (Mat[target][Queue[head]].message);

$\circ$ if the virtual path is not detected and the user is within a branch/interest point area, the app provides information about joining branches, where they take to and about the direction to be taken (e.g. left/right) with respect to the last walked segment of the track (Mat[Queue[head]][Queue[head-1]].message).

- Joystick - Press button stops the current message if a message is being played or provides the overall description of the track otherwise; 
- $\quad$ Slide switch - Up button sends an emergency SMS containing also the current GPS coordinates;

- $\quad$ Slide switch - Down button makes an emergency call.

The application can be applied also in different installation sites or scenarios by simply downloading the specific maps configuration file on the mobile device.

\subsection{The software for the service management}

In order to effectively provide a service for the end-user based on the proposed system, a dedicated software application to manage the pick-up/hand-back of Smart Canes and pre-configured mobile devices was developed. The application is webbased and supports multiple concurrent accesses, via login procedure, to allow the service provider to offer different management offices along the route, where the user can pick-up or hand-back the equipment.

Management offices are usually located in a few points along the path, inside tourist information offices or ticket offices in public buildings (e.g. museums, etc.). The availability of multiple management offices allows visually impaired users to pick-up the equipment in a point of the route, after being registered by a logged in operator, and return them also in a different point of the entire path.

\section{INSTALLATION OF THE SYSTEM ON THE HISTORICAL WALLS OF LUCCA CITY}

During the development period, a short segment of the virtual path was installed for preliminary on-field tests with some final users recruited in collaboration with the Italian Association for the Blind and Visually Impaired - Section of Lucca. The selected site was the pedestrian lane inside the "Baluardo San Donato", on the walls of Lucca city (see Figure 27).

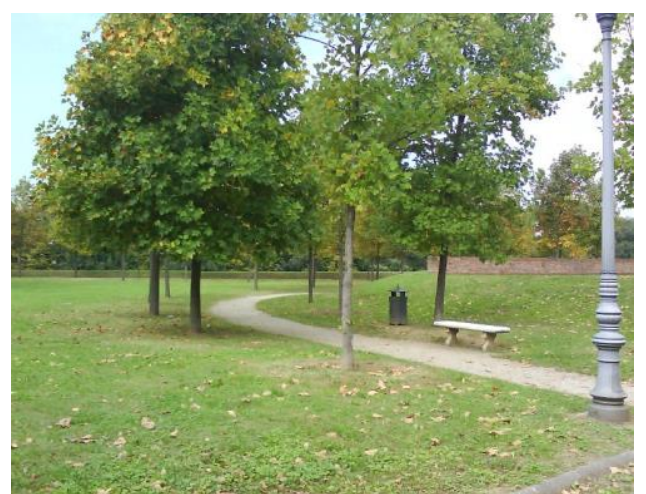

Figure 27. Internal lane on "Baluardo San Donato" used during the preliminary test phase 
The installation phase on the walls of Lucca city started in summer 2013, concurrently with some major paving works on a segment of the pedestrian and bike lane. At the present (July 2018), after two further steps of works, the virtual path has been completely installed on the entire ring on top of the Walls and inside two green areas along the Walls (bastions). The total length is about $4 \mathrm{~km}$.

Figure 28 shows the map of the final installation, highlighting the tracks of the entire path and the location of branch points and points of interest with and without magnetic field cancellation. Additional service points for development purposes are also shown. Figure 29 shows some details of a specific section of the route.

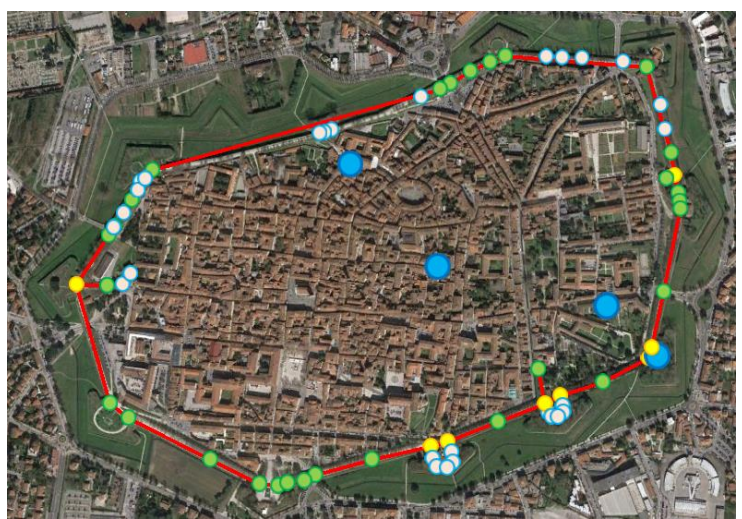

Figure 28. Map of the virtual path installed on the wall of Lucca city. Colored circles represent point of interest, branch points and service points used for development purposes

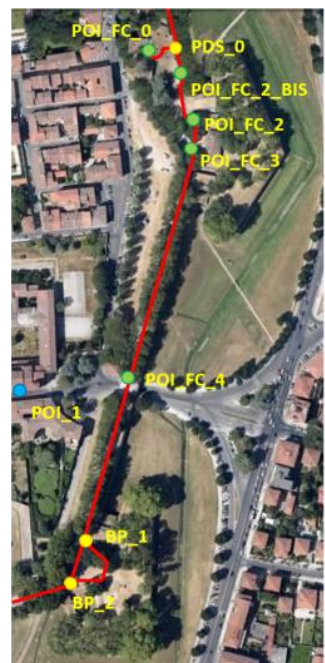

— Virtual path (installed cables)

POI_FC - Point of interest along the route with magnetic field cancellation

BP - Branch point with magnetic field cancellation

- POI - Point of Interest not along the route

Figure 29. Detailed view of a section of the entire route 
Points of interest with magnetic field cancellation, along the main route, are mainly benches aside the path. Points of interest without field cancellation are not along the virtual path and are represented by some buildings of cultural and historical value. Such points may be changed/extended at any time by just modifying the configuration used by the Android application.

Two starting/ending points of the path are foreseen, where the user can pick-up or hand-back the system equipment (i.e. Smart Cane and pre-configured mobile device). They will be located within tourist offices and/or a museum, not far from the lane on top of the Walls. A candidate point is in proximity of the Baluardo San Salvatore; it just faces the main ring on the Walls and is connected to it by a virtual track. Another pick-up/hand-back point is foreseen on the opposite side of the walls, not far from Baluardo San Donato; tracks joining it to the main walking path on the walls are under installation.

Before the installation of the virtual path cables, tests were carried out in order to detect possible path detection problems caused by disturbance signals from other sources. Some sectors of the route showed to suffer from noise coming from the mains supply of the public lighting system, some others from sporadic electrical interference due to for example water pumps near the path or to other unidentified noise sources. This electrical noise picked up by the Path Detector in some points of the walking route is rejected by the SCC microcontroller algorithms that generally avoids false positives (recognition of the presence of the virtual path).

Moreover, before the final installation, GPS logs were taken along the route in order to verify possible critical areas concerning GPS accuracy/availability. GPS reception showed to suffer slightly inside Bastioni and in some areas under trees on the main ring, but it does not represent a major issue for the system.

Finally, Figure 30 shows a schematic view of a virtual path segment installed on the Walls while an example illustrating the user asking the system for information about the surrounding environments is shown in Figure 31.

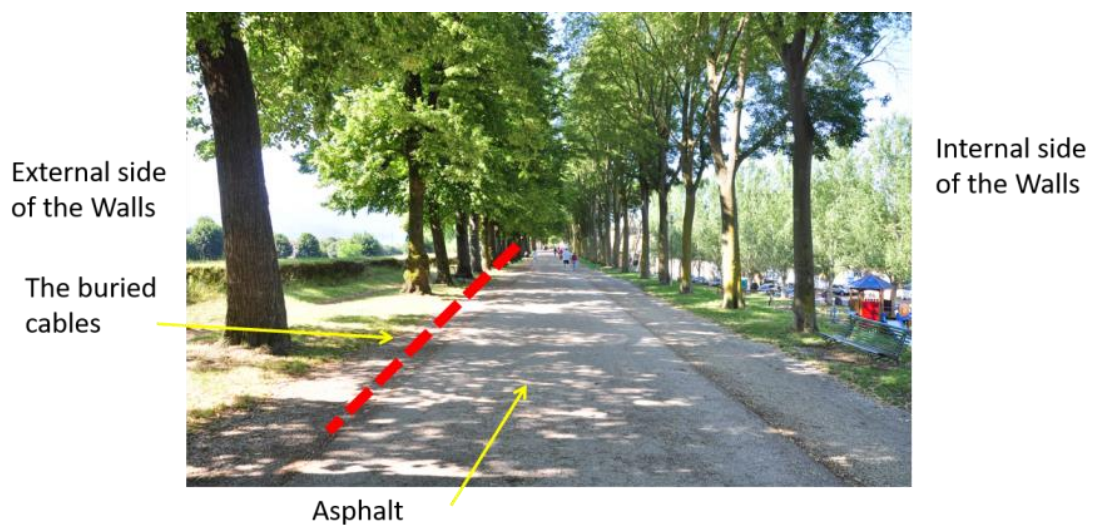

Figure 30. A schematic overview of the path installation on the main route on the walls 


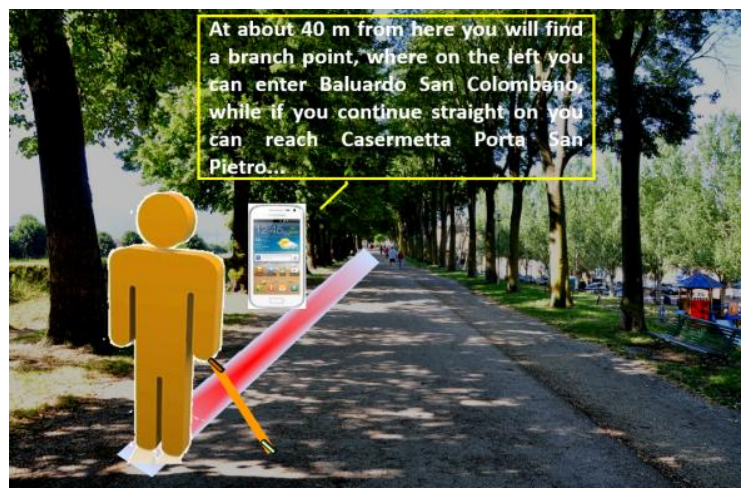

Figure 31. Example of audio message provided by the system during the walk

\section{CONCLUSION}

The proposed mobility aid system for visually impaired people is particularly suited for historical places such as the medieval Walls of the city of Lucca, since it is completely hidden under the terrain. Indeed, the opportunity was provided by a major paving re-work on the pedestrian and bike asphalt road on the walls which was planned in three steps.

The mobility aid system has been already installed and successfully tested with end-users in the first two path segments.

The installation of the last path segment, from a possible pick-up/handback point to the main route, is currently in progress, and is foreseen to be completed by 2018 .

Afterward the facility will be opened to the public. End-users will be able to pick up or hand back the smart-cane and the associated smartphone from one of the starting/ending points of the path. Updated information will be available on the following website: http://www.luccaaccessibile.it/le-mura-per-tutti.

\section{ACKNOWLEDGMENTS}

The project "Le Mura per Tutti" has been partially supported by Fondazione Banca Del Monte di Lucca and Fondazione Cassa di Risparmio di Lucca for the design and development of the hardware/software systems and the installation of the infrastructure on the historical walls.

The authors would like to thank Massimo Diodati (President) and all the friends of the Lucca section of the Italian Association of the Blind and the Visually Impaired for their valuable support in many phases of the project. Thank also to the Municipality of Lucca and Opera delle Mura of Lucca for the support provided, especially during the installation phase. A special thanks to Barbara Leporini, 
Carmen Santoro and Antonello Calabrò of the Italian National Research Council, who developed the very first version of the application on a Symbian-based mobile terminal [34].

Thanks to Luigi Rosi, CEO of the company Veret srl, Capannori (LU), who designed and implemented the fiberglass mechanical structure of the smart cane.

\section{REFERENCES}

[1] Indagine multiscopo sulle famiglie. Condizioni di salute e ricorsi ai servizi sanitari (2004-2005), Istituto Poligrafico e Zecca dello Stato, Rome, 2007 (in Italian)

[2] European Blind Union Newsletter, No 64, September - October 2008. Retrieved from http://euroblindstatic.eplica.is/fichiersGB/nl64.htm

[3] Fanucci L., Roncella R., Iacopetti F., Donati M. and Giannelli N.; A mobility aid system for visually impaired people on the historical walls of Lucca city, Tuscany, Italy; 22nd Mediterranean Conference on Control and Automation; Palermo; 2014

[4] Elmannai W. and Elleithy K.; Sensor-based assistive devices for visuallyimpaired people: Current status, challenges, and future directions; Sensors; 2017

[5] The Cittabile project. (n.a.). Retrieved from http://www.sestosg.net/sportelli/sestoprogetta/agenda21/scheda/1144 (in Italian)

[6] The MAPPED project. (n.a.). Retrieved from http://services.txt.it/MAPPED/index.jsp

[7] Easy Walk. (n.d.). Retrieved from http://easywalk.ilvillage.it/en

[8] Corsair App repository. (n.a.). Retrieved from https://snigle.github.io/corsaire

[9] BlindSquadre website. (n.a.). Retrieved from http://www.blindsquare.com/about

[10] GPS for the visually impaired. (n.a.). Retrieved from https://en.wikipedia.org/wiki/GPS for the visually impaired

[11] Trekker Breeze Plus website. (n.a.). Retrieved from https://store.humanware.com/hau/trekker-breeze-plus-handheld-talkinggps.html

[12] Braille to Note GPS website. (n.a.). Retrieved from https://store.humanware.com/hus/braillenote-gps-software-and-receiverpackage.html

[13] Xiao J., Ramdath K., Losilevish M., Sigh D. and Tsakas A.; A low cost 
outdoor assistive navigation system for blind people; Proceedings of the 2013 8th IEEE Conference on Industrial Electronics and Applications (ICIEA); Melbourne; 2013

[14] Malavasi M., Fanucci L., Evert-Jan H., Iacopetti F., et al; A step forward towards increasing the mobility and participation of people with disabilities utilizing Satellite Navigation Technology Applications;10th European Conference for the Advancement of Assistive Technology in Europe (AAATE); Florence; 2009

[15] Farcy R. and Damaschini R.; Guidance - Assist system for the blind; Laboratoire Aimé Cotton, CNRS Orsay cedex, France

[16] Kwiatkowski P., Jaeschke T., Starke D., Piotrowsky L., Deis H. and Pohl N.; A concept study for a radar-based navigation device with sector scan antenna for visually impaired people; First IEEE MTT-S International Microwave Bio Conference (IMBIOC); Gothenburg; 2017

[17] Ultracane system website. (n.a.). Retrieved from https://www.bel108.it/index.php/apparecchiature-bel/31-ultracane

[18] Safewalk system website. (n.a.). Retrieved from http://www.safewalk.it/it/index.html

[19] Tom Pouce II system website. (n.a.). Retrieved from https://bel108.it/index.php/apparecchiature-bel/3-scheda-tecnica-pollicino

[20] Pollicino Light system website. (n.a.). Retrieved from https://bel108.it/index.php/apparecchiature-bel/14-scheda-tecnicapollicino-ligth

[21] Vítek S., Klima M., Husnik L. and Spirk D.; New possibilities for blind people navigation; Proceedings of the 2011 International Conference on Applied Electronics (AE); Pilsen; 2011

[22] LetiSmart LUCE system website. (n.a.). Retrieved from http://www.letismart.com

[23] Horus system website. (n.a.). Retrieved from https://horus.tech/horus

[24] Brilhault A., Kammoun S., Gutierrez O., Truillet P. and Jouffrais C.; Fusion of artificial vision and GPS to improve blind pedestrian positioning; Proceedings of the 4th IFIP International Conference on New Technologies, Mobility and Security (NTMS); Paris; 2011

[25] Fontanesi S., Frigerio A., Fanucci L. and Li W.; Real-time Pedestrian Crossing Recognition for Assistive Outdoor Navigation; Stud Health Technol Inform; 2015

[26] StepInside system website. (n.a.). Retrieved from https://senion.com/indoor-positioning-system

[27] Li-fi Based Blind Indoor Navigation System for Visually Impaired People. (n.a.). Retrieved from https://www.pantechsolutions.net/projects/li-fi- 
based-blind-indoor-navigation-system-for-visually-impairedpeople\#product tabs description tabbed

[28] Dhruv J.; Path-guided indoor navigation for the visually impaired using minimal building retrofitting; Proceedings of the 16th international ACM SIGACCESS conference on Computers \& accessibility (ASSETS '14); New York; 2014

[29] Biader Ceipidor U., Medaglia C.M., Rizzo F. and Serbanati A.; RadioVirgilio/Sesamonet: an RFID-based Navigation system for visually impaired; 2006

[30] Saaid M.F., Ismail I. and Noor M.Z.H.; Radio frequency identification walking stick (RFIWS): A device for the blind; Proceedings of the 5th International Colloquium on Signal Processing \& Its Applications; Kuala Lumpur; 2009

[31] Tiles for the visually impaired, Loges system. (n.a.). Retrieved from http://www.s-tiles.org/s-

tiles/articoli.nsf/VSNW04E/5F1E432D15468CB0C1256F0E002DAC3D

[32] Tiles for the visually impaired, Apice system. (n.a.). Retrieved from http://www.s-tiles.org/s-

tiles/articoli.nsf/VSNW04E/B5937E10AFED7A80C1256F0E002DAC4C

[33] Guide Dogs for Visually Impaired and Blind People. (n.a.). Retrieved from http://www.livingblind.com/guide-dogs-for-visually-impaired.html

[34] Fanucci L., Roncella R., Iacopetti F., Donati M., Calabrò A., Leporini B. and Santoro C.; Improving mobility of pedestrian visually-impaired users; Proceedings of the 11th European Conference for the Advancement of Assistive Technology (AAATE); Maastricht; 2011 